\title{
ACTIVE TENSION CHANGES IN FROG SKELETAL MUSCLE DURING AND AFTER MECHANICAL EXTENSION*
}

\author{
H. van Atteveldt and A. Crowe $\dagger$ \\ Department of Medical and Physiological Physics, Experimental Physics Laboratory, \\ State University of Utrecht, Princetonplein 5, Utrecht, The Netherlands
}

\begin{abstract}
Isolated frog sartorious muscle at $4^{\circ} \mathrm{C}$ has been used to study the phenomenon whereby tetanically stimulated muscle, subjected to a mechanical extension, yields an active tension which is greater than that obtained during an isometric contraction in which the muscle is stretched prior to stimulation. The excess tension magnitude and decay with time depend upon the total muscle length. After stimulation ceases the excess tension does not persist over a time appreciably longer than that needed for the normal isometric tension to disappear. No persistent excess tension could be seen from stretches applied after stimulation had ceased but before the active tension had fully decayed.
\end{abstract}

Key words - Mechanical properties; sartorius muscle; isometric tension; ramp and hold stretches.

\section{INTRODUCTION}

Investigations carried out on skeletal muscle over the last 20 years have led to the now generally accepted fact that contraction is brought about by the sliding of thin filuments between thick filaments caused by tension exerted by cross-bridges which link the two sorts of filament together (Hansen and Huxley, 1955; Huxley, 1953,1957,1960,1969, 1971, and 1974; Huxley and Niedergerke, 1954; Huxley and Hansen, 1954).

The sliding filament theory can be used to explain the tension-length relation under isometric conditions (Gordon et al, 1966a and 1966b). The developed tension depends upon the amount of effective overlap of the thick and thin filaments and thus upon the number of cross-bridges between them. If the initial length of the muscle is changed prior to stimulation the tension developed fits with the amount of overlap which can be calculated from sarcomere length measurements and ultramicroscopical studies upon the filament lengths. It is known that the lengths of the filaments themselves do not change to any great extent (Harman, 1954; Huxley, 1953; Huxley and Hansen, 1954; Huxley and Niedergerke, 1954).

If, on the other hand, a muscle is stretched during stimulation it would appear that the changes in tension cannot be reconciled with a simple change in overlap of the filaments in spite of the fact (Huxley, 1971) that the bridges are undergoing a continuous make and break process during the active period of the muscle.

When a muscle in tetanic contraction is subjected to a length change of the ramp-and-hold type, the tension change during the ramp part of the stretch depends upon the velocity and extent of the stretch. For the

\footnotetext{
* Received 27 October 1978 ; in revised form 26 January 1979.

† To whom correspondence should be sent.
}

higher range of stretch and velocity values the tension reaches its maximum before the new length is attained and the tension starts to fall while the stretch continucs (Sugi, 1972). This phenomenon has been described as the "slip" phenomenon (Sugi, 1972). It is suggested that after their initial stretching the cross bridges are broken and the speed of the stretch is such that reformation of the cross-bridges cannot take place at a rate sufficient to maintain the tension.

For slower and shorter stretches the tension rises steadily during the ramp part of the stretch, reaches a maximum at the end of the ramp, and then decays during the hold phase (Fowler and Crowe. 1976; Sugi, 1972). It has been reported (Sugi, 1972) that the steady tension attained during the hold part of the stretch is higher than the normal isometric tension at the same length. Sugi (1972) attributes this excess tension to forces exerted by cross-links which are "locked-on" at small displacements. It would appear that the stretching process distorts the cross-links in such a way that they are not spontaneously broken by biochemical processes but can be physically broken due to excessive deformation. However, it is possible that a cross bridge may be of an intermediate length that it is not broken by either of these processes and thus remains in a locked-on tension-exerting state.

However, Sugi (1972) and subsequently other authors (Edman et al., 1976 and 1978; Hill, 1977) performed their experiments on small bundles of fibres. The present paper describes experiments to establish that the excess tension is also seen in whole muscle (frog sartorius). Observations were made upon the magnitude and duration of the effect and preliminary tests are reported to determine what factors such as total muscle length, magnitude and speed of stretch affect the excess tension. Further, investigations are reported in which the stretch is applied to the muscle after the end of the stimulation but before the active tension has completely degenerated. 


\section{MATERIALS AND METHODS}

\section{Preparation}

The experiments were carried out on whole isolated sartorius muscles from Rana pipiens dissected out at the pelvic end with a small piece of bone attached. This was used to attach the preparation to the bottom of the stimulation chamber. The tendon at the distal end of the muscle was held in the vertical and attached to the stretching and tension recording apparatus by means of a short length of light brass chain. The Ringer solution had the following composition $(\mathrm{g} / \mathrm{l}): \mathrm{NaCl}$ 4.24, $\mathrm{KCl}$ 0.148, $\mathrm{MgSO}_{4}$ 0.146, $\mathrm{NaHCO}_{3}$ 2.1, $\mathrm{Na}_{2} \mathrm{HPO}_{4} 0.356, \mathrm{KH}_{2} \mathrm{PO}_{4} 0.068, \mathrm{Na}_{2} \mathrm{SO}_{4} 0.092, \mathrm{Ca}$ gluconate 0.4 , glucose 4.684 . A mixture of $95 \% \mathrm{O}_{2} / 5 \%$ $\mathrm{CO}_{2}$ was continuously bubbled through at such a rate to maintain the $\mathrm{pH}$ at 7.4. $d$.Tubocurarine chloride $(0.01 \mathrm{~g} / 1)$ was added to the Ringer to ensure (by testing on a nerve-muscle preparation) that the muscle was directly stimulated.

\section{Stimulation chamber}

The preparation was mounted vertically in a perspex chamber and at all times remained completely immersed in the Ringer. Stimulation was by stainless steel plates, electrolytically coated with platinum, vertically mounted $15 \mathrm{~mm}$ apart on either side of the muscle. The stimulation pulses from a Grass (S 44) stimulator were fed through a double relay (Devices 2680) connected as a reversing switch so that the current stream between the electrodes was reversed for each alternate pulse to prevent excessive electrolytic effects taking place. The chamber was also provided with a coil of tubing through which cooled water could be circulated to allow experiments to be performed at $4^{\circ} \mathrm{C}$. Most of the experiments were done at this temperature although initially a few were done at room temperature $\left(22^{\circ} \mathrm{C}\right)$. The stimulation chamber was mounted on a table which, by means of a vertical racking device and vernier scale, could be adjusted in height with respect to the muscle stretching device. This allowed the total length of the muscle to be varied and recorded.

\section{Stretching apparatus}

The apparatus was similar to that described previously (Fowler and Crowe, 1976) but modified to provide a vertical stretch. An electromagnetic vibration generator (Pye Ling 403) was used. Movement of its spindle was produced by feeding the signal from a test wave generator (Servomex LF 141) into a current preamplifier the output of which was fed to the vibration generator. The movement was recorded by a Devices 3194 photoelectric displacement transducer and its output was subtracted from the test wave generator signal that was fed to the current amplifier. This provided a servomechanism for the control of the stretching apparatus, the performance of which was improved by inserting a filter into the loop to damp out the natural resonant frequency of the system.
The tension changes were recorded by a Devices (UF 1) load transducer attached to the moving spindle of the vibration generator.

\section{Recording}

Tension and length changes were monitored on an oscilloscope and recorded on a u.v. recorder (S.E. 3006). Data were digitally sampled at $9 \mathrm{msec}$ intervals and fed on-line to a HP $21 \mathrm{MX}$ computer and stored on disk for analysis subsequent to the experiment.

Two Digitimers (Devices D 4030 and 3290) and a Logic Unit (Devices 3080) coupled to each other were used to synchronise the switching of the stimulator and u.v. recorder, to make contact with the computer and to trigger the stretches.

\section{Procedure}

Figure 1 shows how the recordings were used to determine the excess tension. Three recordings were used for each determination as follows:

(1) Isometric contraction. The muscle is held at constant length $A$ and tetanically stimulated at a frequency of 20/sec. This gives tension recording $a$ in Fig. 1.

(2) Contraction with stretch. The muscle was first of all held at the length $A$ for $1 \mathrm{sec}$ to allow initial checks on length and tension to be made. It was then shortened to the new length $B$ and after a further $2 \mathrm{sec}$ stimulation began. During stimulation the muscle was brought back to the length $A$ by means of a ramp-andhold stretch. This is record $b$ in Fig. 1 but the initial period at length $A$ is not included. About $5 \mathrm{sec}$ after stimulation had ceased the length changes $A$ to $B$ and back again to $A$ were repeated. This was done to test that the length changes when the muscle was in the passive state did not bring about tension changes

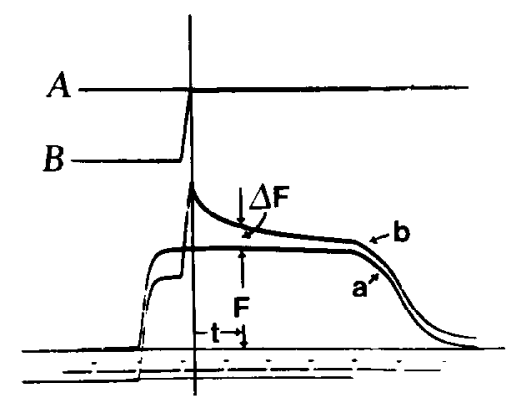

Fig. 1. Sample record to show how excess tension is measured. In this and subsequent similar records, the top horizontal line $A$ gives the fixed length of the muscle for the isometric contractions. The ramp-and-hold stretch $B$ gives the length change for the stimulation with stretch record. The tension development during the isometric contraction is given by $a$ and that during contraction with stretch is given by $b$. The lowest trace gives $1 \mathrm{sec}$ time marks with thereunder a bar to denote duration of stimulation. Three records, two isometric contractions and one contraction with stretch, are superimposed so that the stimulation periods exactly coincide. This allows the differences in tension $\Delta F$ together with the active isometric tension $F$ to be measured at various times $t$ during the hold phase of the stretch. 
which might account for the tension differences observed during stretch and stimulation. These repeat length changes were not recorded on the computer, in the interests of conserving storage space, but were included in the u.v. recorder records.

(3) Repeat of the isometric contraction recording. In the example illustrated in Fig. 1, this tension record is exactly superimposed upon $a$.

A rest interval of at least $5 \mathrm{~min}$ was allowed between each of the recordings. Two isometric contraction records were used to check that any measured tension differences could not arise from a deterioration or change in muscle performance over the intervening rest periods between recordings.

\section{Methods of analysis}

Figure 1 shows how the muscle tensions were compared. The recordings were superimposed so that the periods of stimulation coincided. The excess tension $\Delta F$ and the active tension $F$ in the isometric contraction were measured at various times $t$ from the beginning of the hold phase of the ramp-and-hold stretch. The ratio $\Delta F / F$ we call the percentage excess tension and the on-line computer facilities enabled this to be calculated at $9 \mathrm{msec}$ intervals. The values of $F$ were obtained by taking the means of the two isometric tension recordings.

In certain cases, the values of the percentage excess tension obtained over the hold phase of the stretch were fitted to a multicomponent exponential function in time. For these analyses the DISCRETE program (Provencher, 1976) was used.

\section{RESULTS}

\section{General observations}

In the preliminary experiments it became apparent that several factors i.e. temperature, total muscle length, parameters of the ramp-and-hold stretch, influenced the magnitude of the excess tension. In view of the necessarily long restoration time between successive recordings with respect to the lifetime of the isolated muscle preparation, it was impossible to investigate all the possible combinations of factors on the one muscle. In particular, it was noticed that at room temperature the lifetime of the preparation was even further restricted and it was difficult to obtain consistent consecutive isometric contractions. Therefore no quantitative comparisons of excess tension over different temperatures were made, but it was seen that in general, percentage excess tension was higher at the lower temperature although the magnitude of the isometric tension was lower.

All subsequent experiments were performed at $4^{\circ} \mathrm{C}$ and all results reported here refer to that temperature. This lower temperature had the further advantage that, at the end of tetanic stimulation, the rate of decay of active tension was slowed down. This enabled us to observe how the excess tension continued after stimulation had ceased and also allowed us to try and determine if excess tension appeared if the stretch took place in the interval between end of stimulation and decay of tension to the passive value.

The computer analysis was used to determine the values of the percentage excess tension, particularly after 2 or $3 \mathrm{sec}$ from the beginning of the hold phase. The problem here is that it was often necessary to measure tension differences that were extremely small compared with the total tension that was recorded. Since it was not possible to complctely eliminatc the $50 \mathrm{~Hz}$ from the amplified tension output, the points tended to spread and not lie on a smooth line. It was thus not possible to determine the value of the percentage excess tension at a particular point in time simply by reference to just one data point. The mean of several at the particular time interval must be taken. The computed best fit curve overcame this problem. The values could be found simply by inserting the appropriate time value into the computed equation.

In some of the measurements percentage excess tensions as low as one or two were recorded near the end of the stimulation period. In view of the fact that each measurement consisted of three stimulation periods of the muscle with intervals of several min between each one, it could be argued that these small differences might be due to changes in the tension produced in the muscle itself, perhaps due to gradual fatigue. In such cases we checked for consistency by comparing the contraction with stretch recording, first against the isometric contraction recording preceding it, and then against the isometric contraction that followed, instead of making a comparison with the mean of the two isometric recordings.

\section{Persistence of excess tension after stimulation ceases}

In every case where a measurable percentage excess tension remained at the end of the stimulation period, it was seen that the percentage excess tension actually increased during the first second or so thereafter. While this indicates merely that $\Delta F$ does not decrease so rapidly as $F$ it does not tell us whether $\Delta F$ actually decreases or not. At those shorter muscle lengths where the passive tension was not changed by the mechanical stretch, it was seen that whatever the amount of excess tension remaining at the end of stimulation. the actual tension decayed within a few sec after stimulation ceased to the value that it originally had during the first sec of the record when it was originally at the length $A$.

At those longer muscle lengths where the passive tension changes during stretch, it might be argued that since the passive muscle has visco-elastic properties, it would take some time to settle down to a steady tension after the stretch and this could contribute small tension differences particularly where the active tension has almost decayed to zero. However, in such records it was seen that the tension still decayed nonetheless to its original value within a few $\mathrm{sec}$ of stimulation.

Our general conclusion is thus that although the 

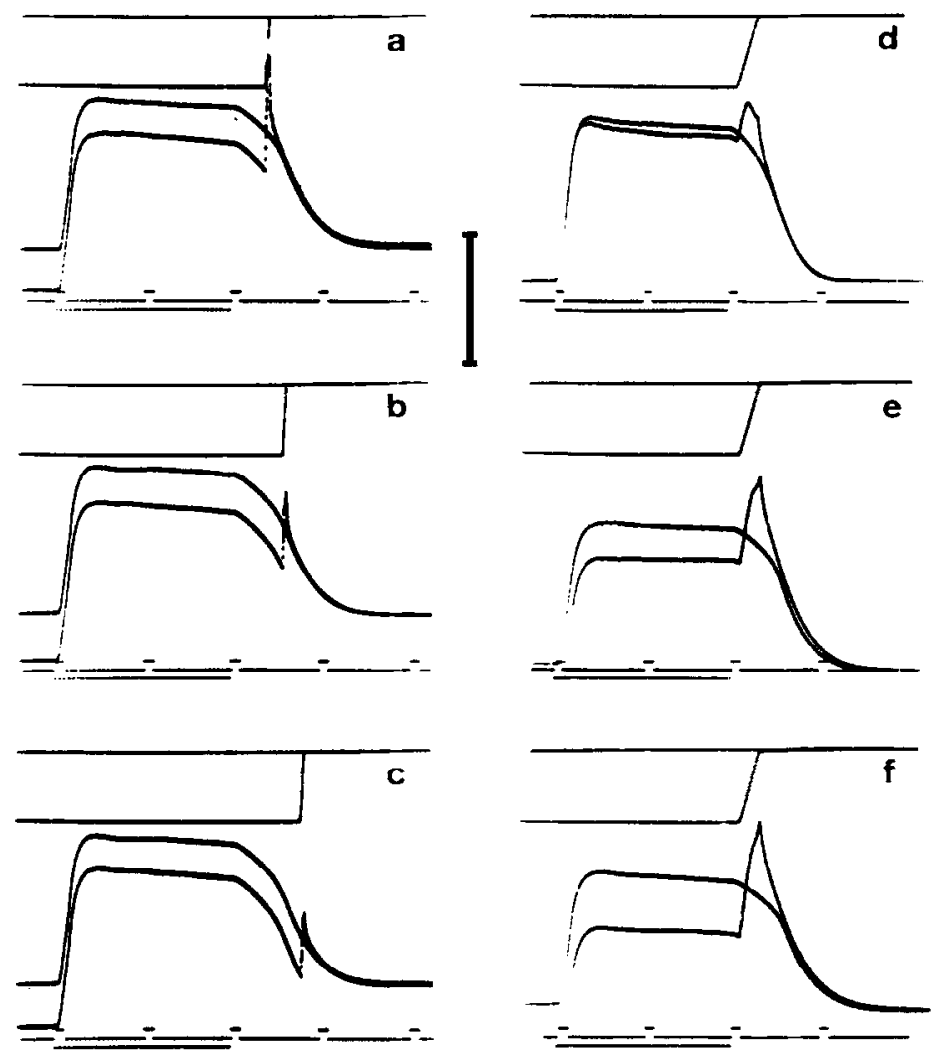

Fig. 2. Tension changes from stretches applied after stimulation has ended. $a, b, c$, records showing identical stretches $(2 \%$ at $40 \% / \mathrm{sec})$ applied at different times after stimulation has ended. The total muscle length was kept constant at $5 \mathrm{~mm}$ greater than the length for maximal isometric tension, $d, e, f$. Identical stretches $(2 \%$ at $8 \% / \mathrm{sec}$ ) applied just subsequent to the end of stimulation but where the muscle was held at three different total lengths, 1, 2 and $5 \mathrm{~mm}$ respectively, in excess of the length for maximal isometric tension. Records show how excess tension rapidly decays and reaches near-zero values within 2 sec from the end of stimulation. Vertical tension bar between $a, d$ represents $0.5 \mathrm{~N}$ and is applicable to each record. It is not possible to compare the total tensions of the muscle in records $d, e, f$ because in each case the position of the oscilloscope beam has been adjusted so that the passive tension (which alters according to the total length) appears at a convenient level on the tube face. Time marks and stimulation bar in $d, e, f$ have been retouched.

excess tension declines more slowly than the active isometric tension, there was no indication that it persisted in any permanent form after stimulation.

\section{Excess tension from stretches applied after stimulation}

In certain experiments, tests were done to study the behaviour of the excess tension when the stretch was applied after stimulation had ceased. Fig. $2 a, b, c$ shows the excess tension for a stretch of $2 \%$ at $40 \% / \mathrm{sec}$ applied at different times after the end of stimulation. Records $2 d, e, f$ show stretches of $2 \%$ at $8 \% / \mathrm{sec}$ applied at the same point in time with respect to the stimulation but for different total muscle lengths. These records are illustrative of the general observation that the excess tension decays fairly rapidly and reaches near-zero values within about $3 \mathrm{sec}$ from the end of stimulation. The percentage excess tension decreased rapidly at the beginning of the hold phase of the stretch and then, after a fraction of a second, began to increase. However it was not considered meaningful to consider values of the percentage excess tension later than about $1 \mathrm{sec}$ after the end of stimulation, in view of the relatively small tensions remaining. Also, if the stretch was applied when the active tension had reached its near-zero value, passive tension changes could also make significant contributions to the tension differences. In order to show that the tension always declines to the same steady value irrespective of when the stretch was applied relative to the end of stimulation, the decay of tension after stretch for five recordings has been plotted in Fig. 3. Here the passive tension prior to stimulation and stretch has been subtracted from the values and the data have been plotted so that zero time in each case corresponds to the beginning of the hold phase of the stretch. The total muscle length was the same in each case and the stretch was always $4.5 \%$ at $72 \% / \mathrm{sec}$. Here it is seen that although the final steady values are the same, they are attained more slowly if the stretch is applied within a short time after stimulation. 


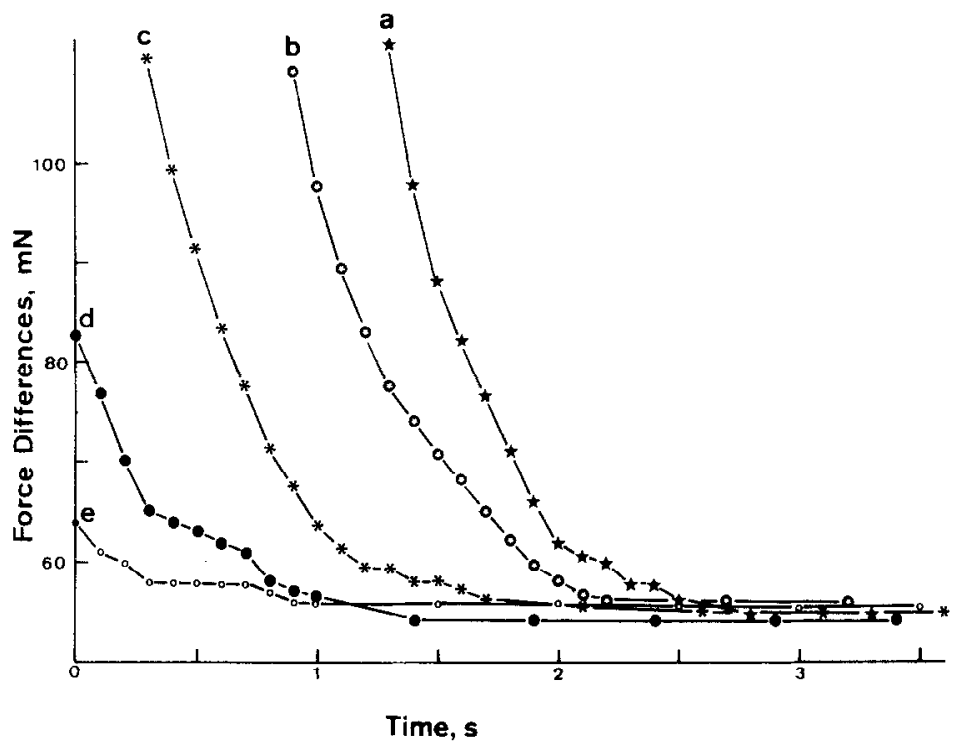

Fig. 3. Force differences obtained from computer data of total muscle tension as a result of stretching at various times after stimulation, and subtracting the passive tension values prior to stimulation and stretch. Graphs plotted so that hold phase of stretch coincides in each case. Amount of stretch $4.5 \%$ at $72 \% / \mathrm{sec}$. Total muscle length, $3 \mathrm{~mm}$ in excess of length for maximal isometric contraction. The stretches began at respectively $0.1,0.4,1.1,1.7$ and $3.0 \mathrm{sec}$ from the end of stimulation for the sequence of records $a-e$. Note how the force difference values always plateau off to the same value, yet this plateau is reached sooner if the stretch is delayed with respect to the end of the stimulation.

In none of the combinations of muscle length, duration and speed of stretch, time from end of stimulation, was there any convincing evidence that the stretch brought about a permanent excess active tension in the muscle that endured beyond the normal isometric tension decay period.

The influence of stretch parameters upon the excess tension

As has been reported by several authors (Fowler and Crowe, 1976; Rack and Westbury, 1974; Sugi, 1972), the form of the tension change during the ramp phase of the stretch depends upon the parameters of the ramp i.e. upon the magnitude and speed of stretch. In particular it is possible to choose a combination where the muscle is so far or so rapidly stretched that no smooth build-up of tension is seen but the maximum tension occurs before the end of the ramp. This is the so-called slip effect (Sugi, 1972). Indeed, it is possible to stretch the muscle so rapidly that the tension falls off to give a negative excess tension at the end of the ramp. We have investigated certain cases where the slip effect was seen but yet the excess tension was still positive at the end of the ramp. Examples are given in Fig. 4. In the two cases where the slip effect is seen, a positive excess tension persisted to the end of stimulation. We formed the impression that the excess tension during the hold phase was dependent upon the amount of excess tension at the beginning of the hold phase irrespective of how the tension built up during the ramp phase. A slight exception to this occurred if a peak in the tension, which preceded a slip, occurred just before the
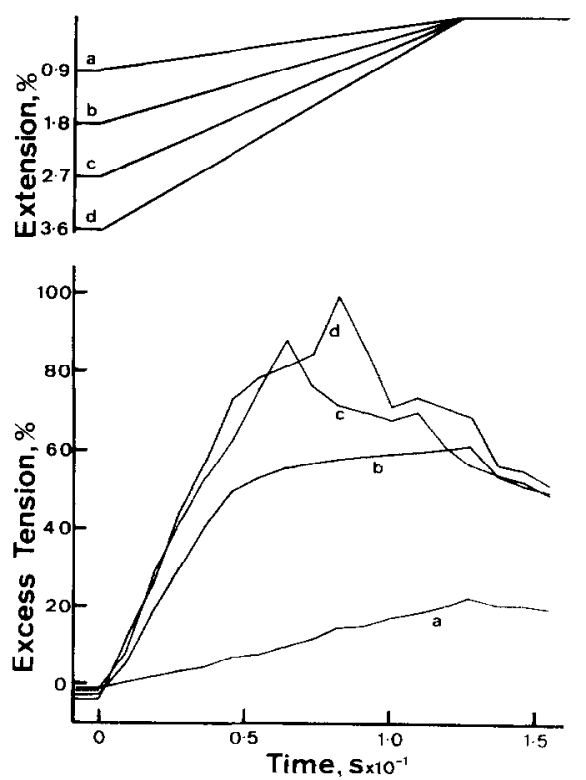

Fig. 4. Computerized values of excess tension obtained at $9 \mathrm{msec}$ intervals for four different ramp heights of equal duration. Here it is seen how the two larger stretches result in peaks which appear before the stretch has ended. The excess tension then falls off to attain values roughly equal that obtained from the second lowest stretch b. $3.5 \mathrm{sec}$ from the beginning of the hold phase the percentage excess tension decayed to $0,3.5,4.5$ and 5.5 for the four stretches $a, b, c, d$. Muscle held at length for maximum isometric contraction. 

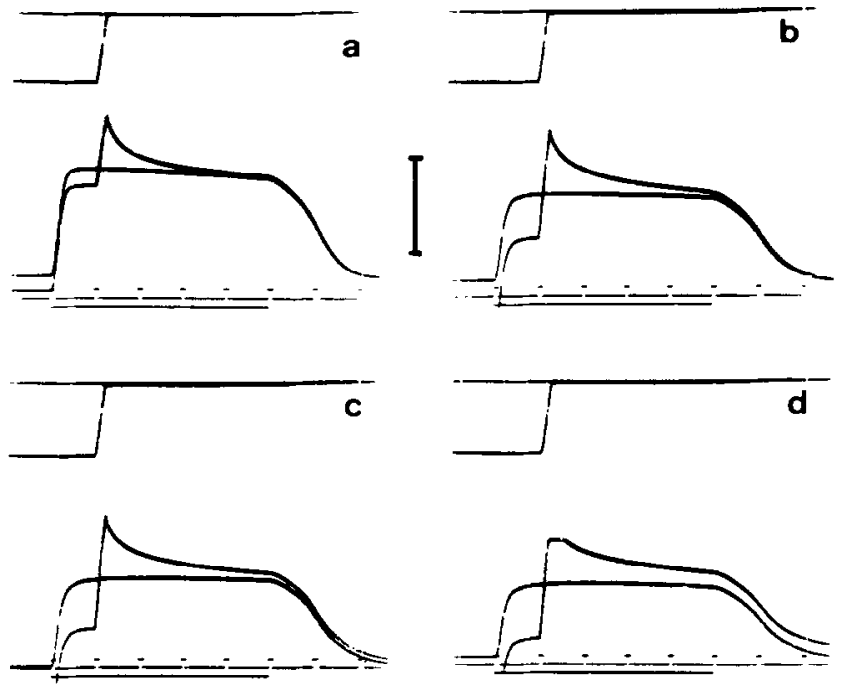

Fig. 5. Effect of variation of total muscle length upon excess tension. Muscle subjected to length change $2 \%$ at $32 \% / \mathrm{sec}$ in each case but the lengths of the muscle, with respect to the length for maximum isometric tension were $a, 0 \mathrm{~mm} ; b, 2.5 \mathrm{~mm} ; c, 3.0 \mathrm{~mm}$ and $d, 4 \mathrm{~mm}$. Vertical bar represents $0.5 \mathrm{~N}$ tension. In each tension record the oscilloscope beam has been adjusted to bring the passive pre-stretch value to a convenient part of the tube face. In $d$ the force transducer went into overload and the peak tension change could not be recorded.

beginning of the hold phase. In such cases the rapid fall-off due to the slip continued into the hold phase to give an initial rather rapid fall-off in excess tension. Apart from this, the excess tension at the end of stimulation was determined by the tension at the beginning of the hold phase.

We did not attempt to study the excess tension subsequent to very rapid stretches which created large slip effects. In such cases, the stretching apparatus did not produce a pure ramp-and-hold. It was felt that the slight overshoot at the beginning of the hold phase would give spurious values of the excess tension.

\section{The effect of total muscle length upon excess tension}

Recordings made at different muscle lengths but for identical stretches are shown in Fig. 5. From these records it is obvious that, in absolute terms, the excess tension is greater for the longer muscle lengths in spite of the fact that the magnitude of the isometric tension is reduced.

Another experiment, illustrated graphically in Fig. 6, shows a tension-length curve for the muscle. Here the tension values were measured $0.5 \mathrm{sec}$ from the beginning of stimulation during the isometric contraction. At each muscle length the excess tension was determined for a $0.5 \mathrm{~mm}$ stretch of duration $0.5 \mathrm{sec}$. These stretch parameters were so chosen that the excess tension rose smoothly to attain its maximum value at the end of the ramp phase. At the shorter muscle lengths up to that which gives the maximum isometric tension, it is seen that the percentage excess tension at the beginning of the hold phase (i.e. $t=0$ ) is reasonably constant, but that for greater muscle lengths it increases. A similar situation is seen when the percentage excess tension is measured at various times

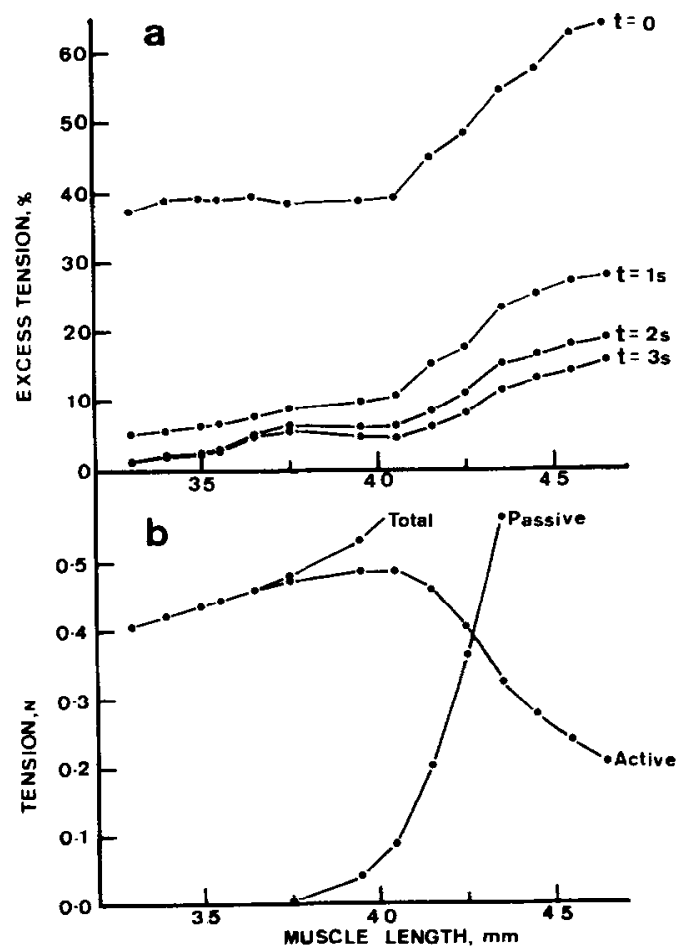

Fig. 6. Tension length diagram of muscle (lower records $b$ ) with corresponding values of percentage excess tension (upper records $a$ ) measured at the beginning of the hold phase and 1,2 and $3 \mathrm{sec}$ subsequently. Stretch $0.5 \mathrm{~mm}$ duration 0.5 sec. Note how the excess tensions at the longer muscle lengths decay more slowly than those for the shorter lengths and how they increase for muscle lengths above that for maximum active tension. 
during the hold phase. Further, it should be noted, as seen from the differences between the curves of Fig. 6 at the respective lengths, that the percentage excess tension decays more rapidly for the shorter muscle lengths than for the longer lengths. In every case however, we were not convinced that the percentage excess tension reached a steady value even after $3.5 \mathrm{sec}$. Certainly for the longer muscle lengths there was no question of a steady value being reached during the period of observation.

Computer analysis of the percentage excess tension into exponential decay terms always yielded a steady component, but this had a lower value than that attained by the experimental values at the end of the analysis period.

Attempts were made, by means of longer stimulation periods, to determine if the excess tension continued to decrease to reach a constant, perhaps zero, value. These were unsuccessful because it was difficult to maintain an isometric tension which either did not fatigue during stimulation or which was sufficiently repeatable to allow several recordings to be made.

\section{DISCUSSION}

Several authors (Edman et al., 1976 and 1978; Hill, 1977; Sugi, 1972) have shown, by using either single fibre preparations or bundles of fibres, that if active muscle is suitably stretched, the tension rises to a peak at the end of the stretch and then decays but maintains a level which is higher than the normal isometric tension at the same length.

The results presented here, obtained from experiments on whole muscle preparations, confirm some of the earlier results. The slip effect is seen if the speed and extent of stretch are sufficiently great. Although much more has to be done to establish under precisely what conditions the slip effect appears, it is noticeable that, in our tests upon whole muscle, it appeared at much lower velocities of stretch than for fibre preparations. This now raises the question as to whether the slip effect ever appears in an in situ muscle. Further tests in this respect clearly need to be carried out since the slip effect would quite clearly be an important consideration when the dynamics of whole joint systems are studied.

As in the case of fibre preparations, we have seen that in whole muscle the excess tension decays during the hold phase and the amount of excess tension is dependent upon the total length of the muscle. However we have not been able to establish, in general, that a plateau value is reached during the hold phase of the stretch even after $3.5 \mathrm{sec}$. Hill (1977) using greater and more rapid stretches maintains that a plateau is reached in tests when the total stimulation time is only $0.6 \mathrm{sec}$. Her experiments were done at room temperature, but even so her results are in good agreement with those of Edman et al. (1976 and 1978) whose preparation was maintained at $0 \cdots 2^{\circ} \mathrm{C}$.
Another point of difference is that we found excess tension persisting at muscle lengths below that for maximum isometric tension as defined by the tensionlength curve. Indeed, in many of our earlier experiments we deliberately set the muscle length so that the muscle was always slack when stretched in the passive state. In such a state the maximal isometric tension was not generated, yet measurable excess tensions could invariably be created and which had not completely decayed by the end of stimulation.

It is difficult to attempt an explanation of these differences between whole muscle and single fibre preparations. Certainly the passive properties differ, but even at the shorter muscle lengths, where the parallel passive component does not make any contribution to the tension changes during a stretch, the decay in excess tension in the whole muscle is slower than that of a single fibre preparation. One is left with the conclusion that the discrepancies are caused by differences in the series elastic connections. In our preparations the muscle was dissected out complete with its tendinous insertions. Tension in the muscle would therefore produce changes in the extension of the series elastic part. As the tension in the muscle decreased, so would the extension of the series elastic part. Therefore, for a constant total muscle length, the contractile part would correspondingly increase in length. This might contribute to an extra excess tension and tend to slow down the decay of excess tension during the hold phase of a stretch.

Clearly the phenomenon of excess tension cannot be explained in terms of actin-myosin overlap as in the sliding filament hypothesis (Gordon et al., 1966a and 1966b; Huxley, 1957). Sugi (1972) suggested that, during the process of stretch, some of the cross bridges are so displaced that they are brought into a "lockedon" situation. They are not biochemically broken by the splitting of ATP but would be broken rapidly by stress forces at larger displacements. Thus in the faster stretches, many of the cross bridges would stretch and be broken, giving rise to the slip effect. The magnitude of the slip effect on the number of cross bridges in the lock-on situation at the end of the stretch would depend upon the speed and magnitude of the stretch. Thus Sugi's idea could account for our observations of the subsequent amount of excess tension depending upon the excess tension at the beginning of the hold phase after slip, if any has taken place. The decay in tension would be due to biochemical breakdown of cross bridges which are stretched, and thus providing extra tension, but not sufficiently stretched to be locked-on.

However, Sugi's idea does not support our finding that the excess tension is not permanent. We have observed the excess tension after stimulation has ended and found no evidence that it remains substantially longer than the decay of a normal isometric tension. Further, our experiments with stretch after stimulation indicate no long-term tension changes.

Thus while it could be argued that stretching a 
muscle may displace some cross bridges so that they have a greater stability and are less readily broken by the biochemical processes, we cannot envisage a situation in which permanent cross bridges exist.

Our experiments confirm previous findings on single fibre preparations with regard to dependence upon total muscle length (Edman et al., 1976 and 1978; Hill, 1977). From Fig. 6 it is seen that the percentage excess tension is fairly constant for muscle lengths up to the value at which the maximum isometric tension takes place. For longer lengths the percentage excess tension steadily increases. This is contrary to what would be expected from the actin myosin overlap pattern if each cross bridge stood an equal chance of being pulled into the locked-on or increased stability state. In that case the percentage excess tension would be constant over the whole range of muscle lengths. The increase in percentage excess tension comes into evidence at those muscle lengths where, by reference to the relationship between tension and actin-myosin overlap (Gordon et al., 1966b), cross bridges can be made at the mid-sarcomere terminals of the actin filaments. At shorter lengths this is excluded by the geometry of the system. During stretch, the terminal cross bridges would be extended out beyond the actin filaments whereas the others would be brought in closer to the actin. If these configuration differences influenced the stability of the cross bridges, i.e. made the terminal cross bridges more stable, then the observed effects could be explained. The percentage excess tension should increase for longer muscle lengths because, as the actin-myosin overlap decreased, the ratio of terminal cross bridges to the total number would increase linearly.

Another possible contributory factor to the dependence of excess tension upon total muscle length could arise from the fact that stretching the muscle at the shorter lengths brings it into a situation where the isometric tension would be higher due to the actinmyosin overlap, whereas stretching it at larger lengths brings it into a situation where the isometric tension would be lower. Since the excess tension is calculated with reference to the isometric tension at the stretched muscle length, we would expect lower values for shorter muscle lengths than for longer muscle lengths provided, of course, that the stretch parameters remained the same.

Although the present experiments have been performed on whole muscle and may perhaps not be so useful in providing extra insight into the contraction mechanism of muscle as experiments performed with a single muscle fibre, the results are indeed useful in terms of the dynamics of joint systems or whole limb movements. In life, muscles are stretched while contracting as often as they shorten (Carlson and Wilkie, 1974). This is particularly true in synergistic pairs of muscle or groups of muscles. The one muscle comes into contraction to arrest motion produced by the other (Valk-Fai and Crowe, 1978; Wadman et al., 1979 and in press). It can be seen from experiments on human arm movement that EMG activity in a brake muscle can start when the limb is in a position where the muscle is in an extended state i.e. its length is greater than that for the creation of maximum tension. The fact that the excess tension created during stretch is greater for longer lengths than for shorter ones suggests that the muscle has an inbuilt mechanism which enables compensatory tension to be created at an unfavourable actin-myosin overlap.

Acknowledgements - The authors acknowledge the critical suggestions from colleagues concerning the manuscript and the help of senior students in the earlier stages of the experimental work.

\section{REFERENCES}

Carlson, F. D. and Wilkie, D. R. (1974) Muscle Physiology. Prentice Hall, Englewood Cliffs, N.I. p. 41.

Edman, K. A. P., Elzinga, G. and Noble, M. I. M. (1976) Force enhancement induced by stretch of contracting single isolated muscle fibres of the frog. J. Physiol., Lond. 258, 95-96.

Edman, K. A. P., Elzinga, G. and Noble, M. I. M. (1978) Further characterization of the enhancement of force by stretch during activity in single muscle fibres of the frog. $J$. Physiol., Lond. 280, 35-36.

Fowler, W. S. and Crowe, A. (1976) Effect of temperature and resistence to stretch of tortoise muscle. Am.J.Physiol. 231, 1344-1355.

Gordon, A. M., Huxley, A. F. and Julian, F. J. (1966a) The tension development in highly stretched vertebrate muscle fibres. J. Physiol., Lond. 184, 143-169.

Gordon, A. M., Huxley, A. F. and Julian, F. J. (1966b) The variation in isometric tension with sarcomere length in vertebrate muscle fibres. J. Physiol., Lond. 184, 170-192.

Hansen, J. and Huxley, H. E. (1955) The structural basis of contraction in striated muscle. Symp. Soc. exp. Biol. 9, 228-264.

Harman, J. W. (1954) Contractions of skeletal muscle myofibrils by phase microscopy. Fedn Proc. Fedn Am. Socs exp. Biol. 13, 430.

Hill, L. (1977) A-band length, striation spacing and tension change on stretch of active muscle. J. Physiol., Lond. 266, 677-685.

Huxley, A. F. (1957) Muscle structure and theories of contraction. Prog. Biophys. biophys. Chem. 71, 255-318.

Huxley, A. F. (1974) Muscular contraction. J. Physiol., Lond. $243,1-43$.

Huxley, A. F. and Niedergerke, R. (1954) Interference microscopy of living muscle fibres. Nature, Lond. 173, 971-973.

Huxley, H. E. (1953) Electron microscope studies of the organisation of the filaments in striated muscle. Biochim. biophys. Acta 12, 387-394.

Huxley, H. E. (1960) Muscle cells. The cell. 4, 365-481. (Edited by Brocket, J. and Minsky, A. E.) Academic Press, London.

Huxley, H. E. (1969) The mechanism of muscular contraction. Science, N.Y. 164, 1356-1366.

Huxley, H. E. (1971) The structural basis of muscular contraction. Proc. R. Soc. B. 178, 131-149.

Huxley, H. E. and Hansen, J. (1954) Changes in the crossstriations of muscle during contraction and stretch and their structural interpretation. Nature, Lond. 173,973-976.

Provencher, S. W. (1976) A Fourier method for the analysis of exponential decay curves. Biophys. J. 16, 27-41.

Rack, P. M. H. and Westbury, D. R. (1974) The short range stiffness of active mammalian muscle and its effect on mechanical properties. J. Physiol., Lond. 240, 331-350.

Sugi, H. (1972) Tension changes during and after stretch in frog muscle fibres. J. Physiol., Lond. 225, 237-252. 
Valk-Fai, T. and Crowe, A. (1978) Analyses of reflex movements in the hind limbs of the terrapin Pseudemys scripta elegans. J. comp. Physiol. A. 125, 351-357.

Wadman, W. J., Denier van der Gon, J. J., Geuze, R. H. and Mol, C. R. (1979) Control of fast goal-directed arm movements. Journal of human movement studies 5, 3-17. Wadman, W. J., Denier van der Gon, J. J. and Derksen, R. J. A. Muscle activation patterns for fast goal-directed arm movements. Journal of human movement studies (in press). 